\title{
The Distribution and Evolution of Black Hole Mass in Broad Line Quasars
}

\author{
Brandon C. Kelly ${ }^{1}$, Marianne Vestergaard ${ }^{2}$, Xiaohui Fan ${ }^{3}$, \\ Lars Hernquist $^{1}$, Philip Hopkins ${ }^{4}$, and Aneta Siemiginowska ${ }^{1}$ \\ ${ }^{1}$ Harvard-Smithsonian Center for Astrophysics, Cambridge, MA, USA \\ ${ }^{2}$ Dark Cosmology Center, Niels Bohr Institute, Denmark \\ ${ }^{3}$ University of Arizona, Tucson, AZ, USA \\ ${ }^{4}$ University of California, Berkeley, CA, USA \\ Email: bckelly@cfa.harvard.edu
}

Keywords. galaxies: active, galaxies: mass function, quasars: general

We present the first estimate of the black hole mass function (BHMF) of broad-line quasars (BLQSOs) that self-consistently corrects for incompleteness and the statistical uncertainty in the mass estimates, based on a sample of 9886 quasars at $1<z<4.5$ drawn from the Sloan Digital Sky Survey. We find evidence for "cosmic downsizing" of black holes in BLQSOs, where the peak in their number density shifts to higher redshift with increasing black hole mass. We estimate the lifetime of the BLQSO phase to be $70 \pm 5 \mathrm{Myr}$ for supermassive black holes (SMBHs) at $z=1$ with a mass of $M_{\mathrm{BH}}=10^{9} M_{\odot}$, and we constrain the maximum mass of a black hole in a BLQSO to be $\sim 10^{10} M_{\odot}$. We find that most BLQSOs are not radiating at or near the Eddington limit. Our results are consistent with models for self-regulated black hole growth, where the BLQSO phase occurs at the end of a fueling event when black hole feedback unbinds the accreting gas.
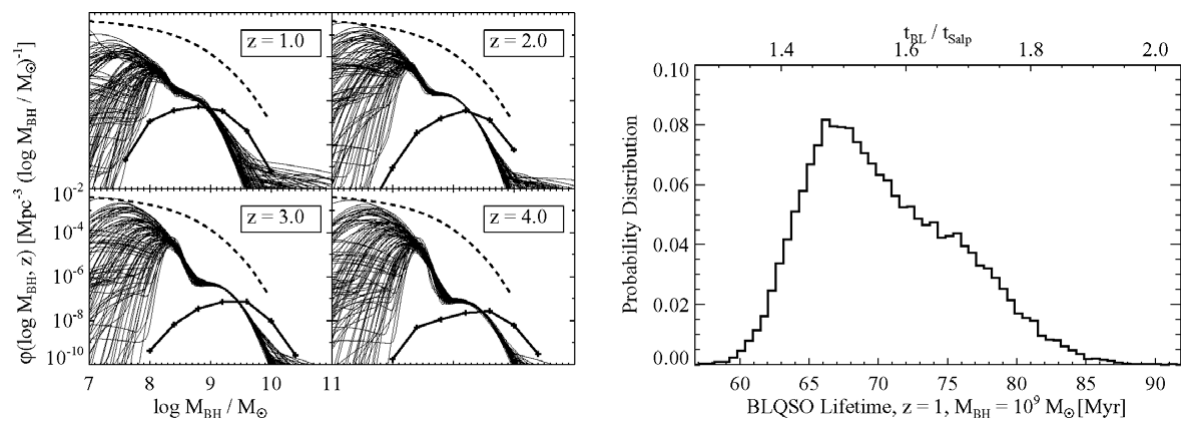

Figure 1. Left: The estimated BHMF for BLQSOs. The thin solid lines are random draws from the probability distribution of the BHMF, the dashed line is the local BHMF for all SMBHs, and the thick solid line is the binned BHMF of the mass estimates from Vestergaard et al. (2008). Right: Probability distribution of the lifetime of the BLQSO phase of $M_{\mathrm{BH}}=10^{9} M_{\odot} \mathrm{SMBHs}$ at $z=1$.

\section{Reference}

Vestergaard, M., Fan, X., Tremonti, C. A., Osmer, P. S., \& Richards, G. T. 2008, ApJ, 674, L1 